\title{
Strumal carcinoid tumour of the ovary presenting with severe constipation
}

\author{
Suzanna Sulaiman ${ }^{1}$, MBChB, MRCOG, Yin Nin $\underline{\text { Chia }}^{2}$, MRCOG, DGO, Ramapadma Vathi Devi Namuduri' ${ }^{2}$, MD, MRCOG
}

\begin{abstract}
A 30-year-old single woman presented with an incidental finding of abdominal mass associated with severe constipation. Her cancer antigen-25, alpha-foetoprotein and beta human chorionic gonadotropin levels were normal, but her carcinoembryonic antigen level was raised at $7.6 \mathrm{~g} / \mathrm{dL}$. Magnetic resonance imaging showed a $11.4 \mathrm{~cm} \times 8.6 \mathrm{~cm}$ $\times 9.5 \mathrm{~cm}$ right ovarian mass with solid and cystic areas. An open right cystectomy was performed. Intraoperatively, she was found to have hirsutism and clitoromegaly. During the operation, there was a right ovarian $10-\mathrm{cm}$ mass, with faecal loading from the caecum to the transverse colon. The uterus, fallopian tubes, left ovary and intraperitoneal survey were normal. Final histology confirmed strumal carcinoid tumour Stage 1A. This case report shows that a strumal carcinoid tumour can present with longstanding constipation as a patient's main complaint and may also be associated with hirsutism.
\end{abstract}

Keywords: constipation, ovary, strumal carcinoid tumour

Singapore Med J 2013; 54(1): e21-e23

\section{INTRODUCTION}

Primary carcinoid tumour of the ovary was first described in 1970. It is rare and regarded as a mesodermal variant of mature teratoma of the ovary. This tumour is characterised by a mixture of carcinoid tissue and thyroid tissue with no intervening struma. Patients can have various presentations with carcinoid tumours. It is commonly unilateral and believed to be easily removed surgically with either a simple oophorectomy or salpingooophorectomy. However, it has been found that patients may present with severe constipation and hirsutism. Here, we report a case reflecting such a presentation.

\section{CASE REPORT}

A 30-year-old single woman was first referred on March 28, 2007 from Tan Tock Seng Hospital (TTSH) to KK Women's and Children's Hospital (KKH). She had an incidental finding of an abdominal mass after being seen for constipation and abdominal distension. The patient had earlier noticed that her trousers were gradually getting tighter over the past two years. This was coupled with a history of severe constipation over the same period, which required chronic use of lactulose to facilitate the passage of bowels on a weekly basis. She denied any history of abdominal weight, loss of appetite or any family history of malignancy. There was no significant past medical history other than gastritis. Incidentally, she also had irregular periods and was a smoker. The initial transabdominal ultrasonography (US) performed at TTSH showed a bulky uterus with a large, round, cystic-solid mass in the body and fundus of the uterus, measuring $6 \mathrm{~cm} \times$ $6 \mathrm{~cm}$. The impression then was that of a generated fibroid. On examination, there was right-sided fullness in the right flank. Vaginal examination was not performed as she was virgo intacta.

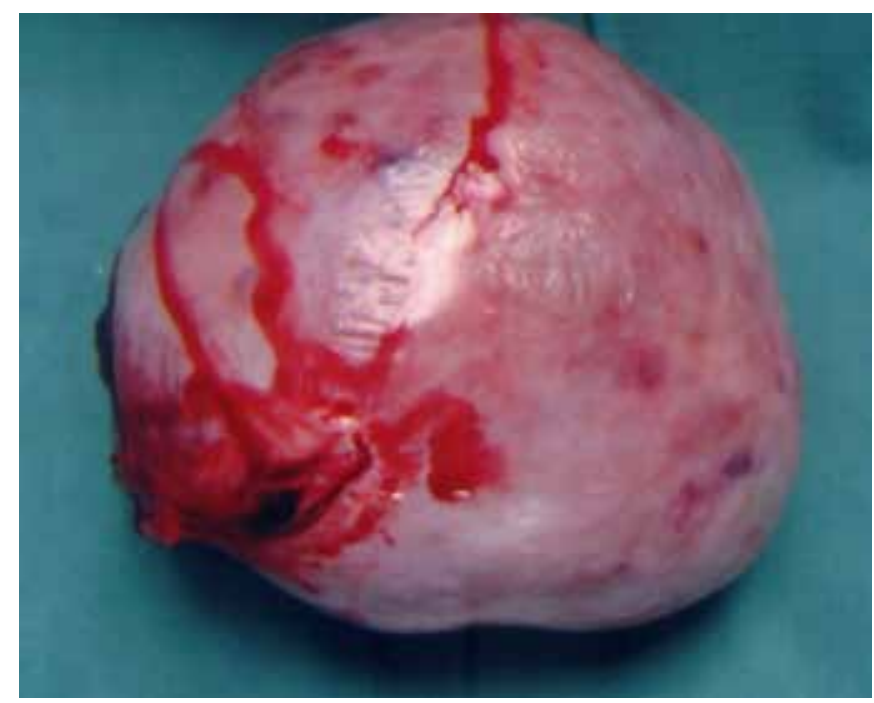

Fig. 11 Photograph shows the gross specimen from oophorectomy of the right ovary.

Repeat pelvic US was arranged at KKH. This revealed a large complex solid/cystic right ovarian mass of about $10 \mathrm{~cm}$. The patient's cancer antigen-25 (CA-125), alpha-foetoprotein (AFP) and beta human chorionic gonadotropin (BHCG) levels were normal, but her carcinoembryonic antigen (CEA) level was raised at $7.6 \mathrm{~g} / \mathrm{dL}$. In view of her history of gastritis, an endoscopy was performed in TTSH, which revealed no abnormalities. Liver function test was also normal. Subsequent magnetic resonance (MR) imaging showed an $11.4 \mathrm{~cm} \times 8.6 \mathrm{~cm} \times 9.5 \mathrm{~cm}$ right ovarian mass with a large, solid cystic mass. There were no liver nodules or ascites.

Consent was obtained for an open right cystectomy, keeping in view a possible oophorectomy with a frozen section of the specimen and staging on June 12, 2007. Intraoperatively, a

${ }^{1}$ Department of Obstetrics and Gynaecology, ${ }^{2}$ Department of Gynaecological Oncology, KK Women's and Children's Hospital, Singapore

Correspondence: Dr Suzanna Sulaiman, Associate Consultant, Department of Obstetrics and Gynaecology, KK Women's and Children's Hospital, 100 Bukit Timah Road, Singapore 229899.suzanna@doctors.org.uk 
10-cm right ovarian mass was seen, while the uterus, fallopian tubes and left ovary were all normal. There was neither ascites nor lymphadenopathy. Intraperitoneal survey was found to be normal. There was faecal loading from the caecum to the transverse colon. Interestingly, the patient was found to have hirsutism and clitoromegaly. The frozen section showed a strumal carcinoid tumour with low-grade malignant features. Right oophorectomy with sparing of the right fallopian tube was thus performed. Final histology confirmed a strumal carcinoid tumour, with the ovarian capsule free of involvement, corresponding with FIGO Stage 1A. Peptide YY (PYY) staining was not performed on the specimen. Peritoneal washings had one cluster of atypical cells, where tumour cells could not be excluded.

In view of the patient's hirsutism and clitoromegaly, postoperative testosterone levels were measured. That, together with thyroid function tests, returned as normal. This case was presented to the multidisciplinary cancer team, and a consensus was reached to observe the patient closely and to conduct a repeat US six months later. The patient had her routine postoperative review on June 25, 2007 and was found to have recovered well with resumption of regular bowel activity.

\section{DISCUSSION}

Carcinoid tumours are derived from cells in the gut wall but can be seen in other organs, including the lungs, mediastinum, thymus, liver, pancreas, bronchis, ovaries, prostate and kidneys. Most carcinoid tumours usually grow slowly and often without any symptoms. A small percentage can have aggressive and metastatic disease (e.g. to the brain). Carcinoid tumour of the ovary is rare and represents less than $0.1 \%$ of ovarian malignancy. ${ }^{(1)}$ It can be primary or metastatic. Patients with such tumours are typically aged 21-77 years, usually with unilateral tumours. The carcinoid component of the strumal carcinoid has been considered a transformation of struma ovarii. . $^{(1,2)}$

Patients with carcinoid tumour may present differently. Depending on the size and location, carcinoid tumours can cause various symptoms, including carcinoid syndrome. Although the presentation is often vague and nonspecific, the symptoms are definitely present. Like our patient, some may present as an incidental finding of an ovarian mass on pelvic US during a routine check-up. As the ovarian mass grows larger, some may complain of abdominal distension. Subsequently, this group may complain of abdominal discomfort or pain as the ovarian mass grows to a significant size. Patients with carcinoid tumours may also present with gastrointestinal symptoms such as constipation or alternating bowel habits. However, only $8 \%$ of the cases exhibited clinical signs of steroid hormone production resulting in endometrial hyperplasia, hirsutism or virilism. ${ }^{(1,2)}$

Treatment with a simple oophorectomy or salpingooophorectomy is usually effective, especially as carcinoid strumal tumour is almost always benign. If the patient opts for a more conservative approach, regular surveillance of the symptoms, size of the cyst, US findings and ovarian tumour markers should be performed. However, if the patient opts for surgical intervention, she must be counselled regarding the two different approaches laparoscopy vs. open laparotomy. This decision would be based on the size of the ovarian mass and the expertise of the surgeon.

Recently, it was reported that strumal carcinoid tumours may be complicated by severe constipation. ${ }^{(3,4)}$ It is very common to find that constipation spontaneously resolves postoperatively. Biologically active substances have been found in carcinoid tumours. These biologically active substances, which are reactive to 17 amine and peptide hormones, could be the cause of constipation rather than being by-products of the mechanical effect of the tumour itself. A study by Motoyama et al illustrated the presence of numerous PYY-positive cells in more than $50 \%$ of all carcinoid tumour cells in all their recruited patients. ${ }^{(3)}$ PYY has the ability to inhibit intestinal motility, which may explain why these patients complained of constipation since such cells were identified in only the carcinoid component of the tumours. ${ }^{(4)}$

Matsuda et al also reported that hormone PYY in the tumour may be the cause of constipation. ${ }^{(1)}$ Like our patient, long-lasting severe constipation completely disappeared after tumour removal. This is again supported in a case report by Shigeta et al, which suggested that the symptoms of constipation resolved once the tumour was removed in their patient. ${ }^{(5)}$ Another case study of a 58-year-old woman with ovarian carcinoid tumour showed that the carcinoid component may trigger two separate functions. ${ }^{(6)}$ Before undergoing surgery, the patient had complained of constipation and hirsutism, and the pathological examination of the ovarian specimen revealed the presence of many carcinoid tumour cells, which were immunohistochemically positive for PYY. ${ }^{(6)}$ Similar to the above studies, this could explain why our patient had complained of constipation. ${ }^{(2)}$ Our patient appears to have many peripheral steroid cells, which may then explain the hirsutism. Immediately after surgery, the patient's constipation resolved. However, despite the decreasing serum level of testosterone, the patient continued to complain of hirsutism for the next two years. It is believed that the ovarian carcinoid tumour has the ability to act on the motility of the bowel and to affect stromal cells. The above studies thus support the belief that the cause of constipation in such patients is the carcinoid component of the tumour. The biologically active substances' effect on intestinal motility may be the main reason. Hence, an abdominal mass, even with a bowel-related complaint, should exclude a gynaecological cause.

In conclusion, this case report shows that strumal carcinoid ovarian tumour should be considered as a differential diagnosis in female patients who present with an abdominal mass that is associated with constipation and hirsutism.

\section{REFERENCES}

1. Matsuda K, Maehama T, Kanazawa K. Strumal carcinoid tumour of the ovary: a case exhibiting constipation associated with PYY. Gynecol Oncol 2002; 87:143-5.

2. Chen CW, Chang WC, Chang DY. Laparoscopic resection of an ovarian strumal carcinoid tumor with dramatic relief of severe constipation. J Minim Invasive Gynecol 2010; 17:242-5. 
3. Motoyama T, Katayama Y, Watanabe H, Okazaki E, Shibuya H. Functioning ovarian carcinoids induce severe constipation. Cancer 1992; 70:513-8.

4. Kawano K, Ushijima K, Fujimato K, Komai K, Kamura T. Peptide $Y Y$ producing strumal carcinoid of the ovary as the cause of severe constipation with contralateral epithelial ovarian cancer. J Obstet Gynecol
Res 2007; 33:392-6.

5. Shigeta $H$, Taga $M$, Kurogi $K$, et al Ovarian strumal carcinoid in severe constipation: immunohistochemical and mRNA analyses of peptide YY. Hum Pathol 1999; 30:242-6.

6. Utsumi N, Hayasako T, Motoyama T. Ovarian carcinoid exhibiting double function. Pathol Int 2003; 53:191-4. 